\title{
Modelling Swimming Hydrodynamics to Enhance Performance
}

\author{
Daniel A. Marinho ${ }^{1,2}$, Abel I. Rouboa ${ }^{3}$, Tiago M. Barbosa ${ }^{2,4}$ and António J. Silva ${ }^{2,5, *}$ \\ ${ }^{1}$ University of Beira Interior, Department of Sport Sciences (UBI, Covilhã, Portugal) \\ ${ }^{2}$ Research Centre in Sports, Health and Human Development (CIDESD, Vila Real, Portugal) \\ ${ }^{3}$ University of Trás-os-Montes and Alto Douro. Department of Engineering (UTAD, Vila Real, Portugal) \\ ${ }^{4}$ Polytechnic Institute of Bragança. Department of Sport Sciences (IPB, Bragança, Portugal) \\ ${ }^{5}$ University of Trás-os-Montes and Alto Douro. Department of Sport, Health and Exercise (UTAD, Vila Real, \\ Portugal)
}

\begin{abstract}
Swimming assessment is one of the most complex but outstanding and fascinating topics in biomechanics. Computational fluid dynamics (CFD) methodology is one of the different methods that have been applied in swimming research to observe and understand water movements around the human body and its application to improve swimming performance. CFD has been applied attempting to understand deeply the biomechanical basis of swimming. Several studies have been conducted willing to analyze the propulsive forces produced by the propelling segments and the drag force resisting forward motion. CFD technique can be considered as an interesting new approach for evaluation of swimming hydrodynamic forces, according to recent evidences. In the near future, as in the present, CFD will provide valorous arguments for defining new swimming techniques or equipments.
\end{abstract}

Keywords: CFD, swimming, evaluation.

\section{INTRODUCTION}

Swimming assessment is one of the most complex but outstanding and fascinating topics in biomechanics. Computational fluid dynamics (CFD) methodology is one of the different methods that have been applied in swimming research to observe and understand water movements around the human body and its application to improve swimming performance. CFD can be considered as a new step forward to the understanding of swimming mechanisms and seems to be an interesting approach to the swimming research.

\section{BACKGROUND OF CFD METHODOLOGY}

CFD is a branch of fluid mechanics that solves and analyses problems involving a fluid flow by means of computer-based simulations. CFD methodology consists of a mathematical model that replaces the Navier-Stokes equations with discretized algebraic expressions that can be solved by iterative computerized calculations. The NavierStokes equations describe the motion of viscous noncompressible fluid substances. These equations arise from applying Newton's second law to fluid motion, together with the assumption that the fluid stress is the sum of a diffusing viscous term (proportional to the gradient of velocity), plus a pressure term. A solution of the Navier-Stokes equations is called a velocity field or flow field, which is a description of

*Address correspondence to this author at the Department of Sport Sciences, University of Trás-os-Montes and Alto Douro, Vila Real, Portugal, Rua Dr. Manuel Cardona, 5000 Vila Real Portugal;

Tel: +351 259350000; E-mail: Ajsilva@utad.pt the velocity of the fluid at a given point in space and time. CFD methodology is based on the finite volume approach. In this approach the equations are integrated over each control volume. It is required to discretize the spatial domain into small cells to form a volume mesh or grid, and then apply a suitable algorithm to solve the equations of motion. In addition, CFD analyses complements testing and experimentation, reducing the total effort required in the experimental design and data acquisition.

In the beginning of its application CFD was quite difficult to use. It was applied only in a few companies of high technological level, namely in the Aerospatiale Engineering or in some specific scientific research areas. It became obvious that its application had to assume a user friendly interface and to progress from a heavy and difficult computation to practical, flexible, intuitive and quick software. Therefore, the following step was to transform CFD in a new set of commercial software to be used in different applications and to help the connection between the user and the computer.

Presently, this tool is used in the resolution of complex engineering problems involving fluid dynamics and it is also being extended to the study of complex flow regimes that define the forces generated by species in self propulsion.

The basic steps of CFD analysis are:

1. Problem identification and pre-processing: (i) define the modelling goals, (ii) identify the domain that wants to model, (iii) design and create the grid.

2. Solver execution: (i) set up the numerical model, (ii) compute and monitor the solution. 
3. Post-Processing: (i) examine the results; (ii) consider revisions to the model.

\section{ADVANTAGES AND LIMITATIONS OF CFD}

CFD can be used to predict fluid flow, heat and mass transfer, chemical reactions and related phenomena by solving the set of governing mathematical equations. The results of CFD analyses can be relevant in conceptual studies of new designs, detailed product development, troubleshooting and redesign.

Lyttle and Keys [1] referred that CFD can provide the answers into many complex problems which have been unobtainable using physical testing techniques. One of the major benefits is to quickly answer many "what if" type questions. It is possible to test many variations until one arrives at an optimal result, without physical/experimental testing. CFD could be seen as bridging the gap between theoretical and experimental fluid dynamics. For example, with this methodology it is possible to study the aerodynamic of a race car before being constructed or to study the air flow inside the ventilation system of a park station, to simulate situations where a fire takes place, to analyse the ventilation and the acclimatisation of a specific building, such as an hospital where the quality of the air is quite important.

CFD was developed to model any flow filed provided the geometry of the object is known and some initial flow conditions are prescribed. CFD is based on the use of computers to solve mathematical equation systems. However, it is essential to apply the specific data to characterize the study conditions. Therefore, in the CFD studies the subject who analyzes the problem must be considered. The scientific knowledge, the computational program which solves the equations system representing the problem, the kind of computer that executes the defined calculations in the numerical program and the person who verifies and analyses the obtained results must also be taken in account.

In this sense, one must consider that the CFD analyses can have some inaccurate results if there is not thorough study of the specific situation. The inserted data should not have wide-ranging estimation. On the other hand, the available computational resources can be insufficient to obtain results with the necessary precision. Previous to any simulation, the flow situation must be very well analysed and understood, as well as of the obtained results.

\section{VALIDITY, RELIABILITY AND ACCURACY OF CFD}

CFD studies are becoming more and more popular. However, a main concern still persists. Can the numerical data be comparable with experimental research? Are the numerical results accurate enough to be meaningful and therefore have ecological validity? For sport scientists who work in close connection with coaches and athletes this question is important in order to give good, appropriate and individual feedbacks for practitioners.

Several studies within different scopes attempted to verify the validity and accuracy of CFD. This numerical tool has been validated as being feasible in modelling complicated biological fluid dynamics, through a series of stepwise baseline benchmark tests and applications for realistic mod- elling of different scopes for hydro and aerodynamics of locomotion [2].

In bioscience, Yim et al. [3] described in detail critical aspects of this methodology including surface reconstruction, construction of the volumetric mesh, imposition of boundary conditions and solution of the finite element model. Yim et al. [3] showed the validity of the methodology in vitro and in vivo for experimental biology. Barsky et al. [4] have also demonstrated good agreement between the numerical and experimental data on tethered DNA in flow. Moreover, Gage et al. [5] reported that computational techniques coupled with experimental verification can offer insight into model validity and showed promise for the development of accurate three-dimensional simulations of medical procedures.

In engineering, Venetsanos et al. [6] illustrated an application of CFD methods for the simulation of an actual hydrogen explosion occurred in a built up area of central Stockholm (Sweden) in 1983. The subsequent simulation of the combustion adopted initial conditions for mean flow and turbulence from the dispersion simulations, and calculated the development of a fireball. This data provided physical values that were used as a comparison with the known accident details to give an indication of the validity of the models. The simulation results were consistent with both the reported near-field damage to buildings and persons and with the far-field damage to windows.

In sports some trials have been carried-out to compare the numerical results with experimental results also. A combined CFD and experimental study on the influence of the crew position on the bobsleigh aerodynamics was conducted by Dabnichki and Avital [7]. The experimental results obtained in a wind tunnel suggested that the adopted computational method is appropriate and yields valid results. In what concerns to aquatic sports there is a lack of studies comparing experimental and CFD data. However, CFD was developed to be valid and accurate in a large scope of fluid environments, bodies and tasks, including sports. So, it is assumed that CFD have ecological validity even for swimming research.

Another important concern is related with CFD reliability. In experimental tests, the input data are not always the same and thus the outputs will vary. However, the numerical simulations allow having always the same input conditions and therefore the same outputs.

\section{CFD IN SWIMMING: PRACTICAL CONCERNS}

CFD has been applied attempting to understand deeply the biomechanical basis of swimming. Several studies have been conducted willing to analyze the propulsive forces produced by the propelling segments [e.g. 8,9] and the drag force resisting forward motion [e.g. 10,11].

Regarding the propelling forces in swimming, the main CFD results pointed out that:

1. The drag coefficient was the main responsible for the hand and forearm propulsion, with a maximum value of force corresponding to an angle of attack of $90^{\circ}[12,13]$.

2. An important contribution of the lift force to the overall force generation by the hand/forearm in swimming 
phases was observed with angles of attack of $30^{\circ}, 45^{\circ}$ and $60^{\circ}$, especially when the little finger leads the motion [12].

3. The hand model with the thumb adducted presented higher values of drag coefficient compared with thumb abducted models. The model with the thumb fully abducted allowed increasing the lift coefficient of the hand at angles of attack of $0^{\circ}$ and $45^{\circ}$ [13].

4. The resultant force coefficient showed that the hand model with the thumb fully abducted presented higher values than the positions with the thumb partially abducted and adducted at angles of attack of $0^{\circ}$ and $45^{\circ}$. At an angle of attack of $90^{\circ}$, the model with the thumb adducted presented the highest value of resultant force coefficient [13].

5. The hand model with little distance between fingers presented higher values of drag coefficient than the models with fingers closed and fingers with large distance spread. The values for the lift coefficient presented little differences between the models with different finger's spreading [14].

6. The results suggested that for hand positions in which the lift force can play an important role, the abduction of the thumb may be better, whereas at higher angles of attack, in which the drag force is dominant, the adduction of the thumb may be preferable. Furthermore, it is suggested that fingers slightly spread could allow the hand to create more force during swimming [13,14].

Regarding the drag forces resisting forward motion, the main results pointed out that:

1. A queue distance of $6.00 \mathrm{~m}$ may cause in the back swimmer a drag force of $\approx 84 \%$ of that of the leading swimmer [15].

2. As a suggestion to specific swimming training sets, the back swimmer must start swimming at least when the leading swimmer reaches a $10 \mathrm{~m}$ distance from the starting wall, rather than the $5 \mathrm{~m}$ distance commonly used in training. This distance will allow both swimmers to be in the same hydrodynamic conditions [15].

3. Concerning open water competitions, the athletes could take important advantages of swimming in a drafting situation [15].

4. Concerning the gliding phases after the starts and turns, it seemed that for velocities higher than $2.40 \mathrm{~m} / \mathrm{s}$ there is a trend for the small/fast kick to become more effective whereas for velocities lower than $2.40 \mathrm{~m} / \mathrm{s}$ the large/slow kick appeared to be more effective [1].

5. However, at higher velocities it seemed more efficient to the swimmer to maintain a streamlined position than to initiate underwater kicking. The authors stated that this situation is due to the swimmer creating more active drag than propulsion while kicking compared to remaining in a streamlined position, thus leading to a negative acceleration of the swimmer. Although, it appeared that the swimmer would benefit from a smaller kick at higher velocities, it seemed better to maintain a streamlined position [1].
6. The gliding position with the arms extended at the front, with the shoulders flexed, presented lower drag coefficient values than the position with the arms placed along the trunk. Considering the breaststroke turn, Marinho et al. [11] suggested that the first gliding, performed with the arms at the front, should be emphasized in relation to the second gliding, performed with the arms along the trunk.

7. The position of the head had a noticeable effect on the hydrodynamic performances, strongly modifying the wake around the swimmer. The position with the head aligned with the body seemed to allow the swimmer to carry out the best water penetration during the underwater swimming phases, comparing with a lower and a higher head position. The head aligned with the axis of the body induces a decrease in the drag from $17 \%$ to $21 \%$, for a range velocity from $2.20 \mathrm{~m} / \mathrm{s}$ to $3.10 \mathrm{~m} / \mathrm{s}$ [16].

A topic to be developed in the future is the analysis of equipments and facilities using CFD. The repercussion of training equipments (e.g., fins, boards, paddles, pull-buoys) in swim technique, as well, as the swim wear itself (e.g., swim suits, caps, goggles) and facilities (e.g., swim lane design, water flows at the surface and in the bottom of the swimming pool, materials and geometry of head and lateral walls) in performance are an outstanding research opportunity.

\section{CONCLUSION}

In summary CFD technique can be considered as an interesting new approach for evaluation of swimming hydrodynamic forces, according to recent evidences. In the near future, as in the present, CFD will provide valorous arguments for defining new swimming techniques or equipments.

\section{REFERENCES}

[1] Lyttle A, Keys M. The application of computational fluid dynamics for technique prescription in underwater kicking. Port J Sport Sci 2006; 6(Suppl 2): 233-5.

[2] Liu H. Computational biological fluid dynamics: digitizing and visualizing animal swimming and flying. Integr Comp Biol 2002; 42: 1050-9.

[3] Yim P, Demarco K, Castro MA, Cebral J. Characterization of shear stress on the wall of the carotid artery using magnetic resonance imaging and computational fluid dynamics. Stud Health Technol Inform 2005; 113: 412-42.

[4] Barsky S, Delgado-Buscalioni R, Coveney PV. Comparison of molecular dynamics with hybrid continuum-molecular dynamics for a single tethered polymer in a solvent. J Chem Phys 2004; 121 : 2403-11.

[5] Gage KL, Gartner MJ, Burgreen GW, Wagner WR. Predicting membrane oxygenator pressure drop using computational fluid dynamics. Artif Organs 2002; 26: 600-7.

[6] Venetsanos AG, Huld T, Adams P, Bartzis JG. Source, dispersion and combustion modelling of an accidental release of hydrogen in an urban environment. J Hazard Mater 2003; 105(1-3): 1-25.

[7] Dabnichki P, Avital E. Influence of the position of crew members on aerodynamics performance of two-man bobsleigh. J Biomech 2006; 39: 2733-42.

[8] Bixler B, Riewald S. Analysis of swimmer's hand and arm in steady flow conditions using computational fluid dynamics. J Biomech 2002; 35: 713-7.

[9] Rouboa A, Silva A, Leal L, Rocha J, Alves F. The effect of swimmer's hand/forearm acceleration on propulsive forces generation using computational fluid dynamics. J Biomech 2006; 39: 1239-48. 
[10] Bixler B, Pease D, Fairhurst F. The accuracy of computational fluid dynamics analysis of the passive drag of a male swimmer. Sports Biomech 2007; 6: 81-98.

[11] Marinho DA, Reis VM, Alves FB, et al. The hydrodynamic drag during gliding in swimming. J Appl Biomech 2009; 25(3): 253-7.

[12] Silva AJ, Marinho DA, Reis VM, et al. Study of the propulsive potential of the hand and forearm in swimming. Med Sci Sports Exerc 2008; 40(5 Suppl.): S212.

[13] Marinho DA, Rouboa AI, Alves FB, et al. Hydrodynamic analysis of different thumb positions in swimming. J Sports Sci Med 2008; 8: 58-66.
[14] Marinho DA, Barbosa TM, Reis VM, et al. Swimming propulsion forces are enhanced by a small finger spread. J Appl Biomech 2008 (in press).

[15] Silva AJ, Rouboa A, Moreira A, et al. Analysis of drafting effects in swimming using computational fluid dynamics. J Sports Sci Med 2008; 7(1): 60-66.

[16] Zaidi H, Taiar R, Fohanno S, Polidori G. Analysis of the effect of swimmer's head position on swimming performance using computational fluid dynamics. J Biomech 2008; 41: 1350-8.

(C) Marinho et al.; Licensee Bentham Open.

This is an open access article licensed under the terms of the Creative Commons Attribution Non-Commercial License

(http://creativecommons.org/licenses/by-nc/3.0/) which permits unrestricted, non-commercial use, distribution and reproduction in any medium, provided the work is properly cited. 